\title{
Stance control is not affected by paresis and reflex hyperexcitability: the case of spastic patients
}

\author{
A Nardone, M Galante, B Lucas, M Schieppati
}

Institute of Human Physiology, University of Pavia, Via Forlanini 6, I-27100 Pavia, Italy, and Salvatore Maugeri Fondation (IRCCS), Pavia, Italy

M Schieppati

Salvatore Maugeri Foundation (IRCCS), Division of Physical Therapy and

Rehabilitation, Posture and Movement

Laboratory, Scientific

Institute of Veruno,

Novara, Italy

A Nardone

M Galante

Groupe d'Etudes et de la Recherche sur le Handicap (GERSH), Centre de

Convalescence et de Rééducation,

Université de

Bourgogne, Dijon,

France

B Lucas

Correspondence to:

Professor M Schieppati,

marco.schieppati@unipv.it

Received 13 June 2000 and in revised form

11 December 2000

Accepted 5 January 2001

\begin{abstract}
Objectives-Spastic patients were studied to understand whether stance unsteadiness is associated with changes in the control of voluntary force, muscle tone, or reflex excitability, rather than to abnormal posture connected to the motor deficit itself.
\end{abstract}

Methods-Twenty four normal subjects, 12 patients affected by amyotrophic lateral sclerosis (ALS), seven by spastic paraparesis, and 14 by hemiparesis were studied. All patients featured various degrees of spasticity and paresis but were free from clinically evident sensory deficits. Body sway during quiet upright stance was assessed through a stabilometric platform under both eyes open (EO) and eyes closed (EC) conditions. The sudden rotation of a supporting platform, in a toe up and toe down direction respectively, evoked short (SLR) and medium latency (MLR) reflex responses to stretch of the soleus or the tibialis anterior (TA) muscle. Results-No relation was found between clinical findings (tone, muscle strength, tendon reflexes, plantar response, and duration of disease) and body sway. On average, all patient groups exhibited a forward shift of the centre of foot pressure (CFP) with respect to normal subjects; in addition, paraparetic and to a much larger extent hemiparetic patients showed a lateral shift of CFP. Body sway area was significantly increased only in the hemiparetic patients. No relation was found between position of the CFP and sway within any patient group. Soleus SLR was increased in all patients with respect to normal subjects. TA SLR was often seen in both patients with ALS and paraparetic patients, but only rarely in normal subjects and hemiparetic patients. However, no relation was found between amplitude of soleus or TA SLRs and stabilometric variables. The frequency and size of soleus MLR and TA MLR were decreased in all patients. These responses were decreased in size and not modulated by background EMG in the affected leg of hemiparetic patients, suggesting a disturbed control of spinal reflexes fed by spindle group II afferent fibres.

Conclusions-It is proposed that body posture, paresis, or monosynaptic reflex hyperexcitability do not affect the control of equilibrium during quiet upright stance. In hemiparetic patients, the decreased amplitude of MLRs might be the main cause of the large postural instability. The results are congruent with the hypothesis of a role for group II afferent input in the reflex control of equilibrium. (F Neurol Neurosurg Psychiatry 2001;70:635-643)

Keywords: spasticity; stance; body sway; reflex responses

The control of human upright stance relies on multiple sensory inputs, their central integration, and appropriate reflex and voluntary motor output. Despite many studies on the role of afferent input in the control of body sway during quiet stance, ${ }^{1}$ an analysis of the effects produced by lesions of the motor system has not been systematically pursued. Patients with cerebrovascular accidents sway to a larger extent than normal subjects ${ }^{2}$; however, it is not clear whether unsteadiness is a direct consequence of the motor deficit itself (decrease in muscle strength, asymmetric support) or of the changes in the control of voluntary force or muscle tone and reflex excitability, accompanying the physiological changes in the central organisation of motor activity.

Reportedly, changes in muscle postural tone and stretch reflex excitability can produce abnormal sway. ${ }^{4}$ On the other hand, recent results from this laboratory have shown that patients affected by large fibre peripheral neuropathy do not necessarily present with increased body sway despite decreased muscle force and absence of tendon reflex. ${ }^{5}$ In the present investigation, we analysed the characteristics of quiet upright stance in a group of spastic patients affected by various lesions of the CNS. These lesions have in common alterations of descending motor pathways leading to decreased muscle force and increased muscle tone and spinal reflex excitability. Contrary to polyneuropathic patients, spastic patients show hyperactive tendon jerks. To check the possibility that changes in stretch reflex excitability produce abnormal sway, we analysed latency and amplitude of the reflex responses evoked in the leg muscles by perturbations of upright posture delivered by a mobile platform.

Although the input from the skin receptors of the foot sole may contribute to the control of stance in normal subjects, ${ }^{6}$ no information exists about the possible effects on stance of an abnormal central processing of the cutaneous input. In spastic patients, such abnormality is witnessed by the reversed sign of the cutaneous plantar response, which occurs as a consequence of upper motor neuron lesions. ${ }^{7}$ Therefore, we 
considered the relation between sway and the Babinski sign as well.

A further cause of changes in body sway in spastic patients might be their abnormal postural attitude, as sway amplitude depends on the anterior-posterior position of the projection of the body centre of mass on the base of support in normal subjects. ${ }^{8-10}$ Therefore, a second aim of this study was to check the possible dependence of increased body sway on peripheral conditions, such as degree or asymmetry of muscle strength and abnormal position of body centre of mass, typical sequelae in hemiparetic patients.

\section{Methods}

PATIENTS

The study was performed on 33 spastic patients and 24 normal subjects. The patient group was characterised as follows: 12 patients with sporadic amyotrophic lateral sclerosis (ALS), mean age 56.9 years (SD 10.1) (range 39-73), seven with spastic paraparesis, mean age 53.1 (SD 10.7) (range 39-67), and 14 with stroke, mean age 56.6 (SD 13.5) (range 29-80). Although the onset of ALS was apparently localised at spinal level, a variable degree of upper motor neuron syndrome was present. Four paraparetic patients were affected by Strümpell-Lorrain disease, two by idiopathic spastic paraparesis, and one by sequelae of removal of meningioma of the falx cerebri. Eight of the hemiparetic patients showed right hemiparesis, six left hemiparesis. In three, the lesion was localised at the cortical grey matter, whereas in the other 11 the lesion involved also the subcortical white matter, mainly in the territory of the medial cerebral artery. In no patient was the right posterior parietal cortex, which is involved in the processing of spatial information, ${ }^{11}$ affected by the vascular lesion. All patients were deliberately recruited only if free from clinical sensory deficits. Mean duration of disease was 1.4 (SD 1.0), 11.0 (SD 10.1), and 0.7 (SD 0.8) years for patients with ALS, and paraparetic and hemiparetic patients, respectively. Patients were able to stand upright and walk without aids. All were enrolled in a rehabilitation programme aimed at improving gross motor function (rolling, sit to stand, transferring), upper limb function, and gait. Bobath techniques were utilised. Due to the different types of disease, patients entered the study at different times from the onset of disease. As a consequence, some patients were enrolled some weeks after rehabilitation, other patients at the start. The control group was made up of a sample of 24 subjects, mean age 58.6 years (SD 12.1) (range $41-83$ ). They had never had metabolic, neurological, or vestibular disease, did not take drugs known to affect the CNS, and their present neurological examination was unremarkable. All subjects and patients gave their informed consent to the protocol, and the local ethics committee approved the study.

CLINICAL EXAMINATION

Muscle strength and tone, tendon reflexes, and plantar response were clinically assessed in the lower limbs by means of a standard neurological investigation. Tone of foot plantarflexor muscles was graded using the modified Ashworth scale ${ }^{12}{ }_{13}$ from 0 (no increase in muscle tone) to 5 (impossible to flex or extend the ankle). For scoring muscle strength, a motricity index of ankle dorsiflexion derived from the Medical Research Council grades was used. ${ }^{14}$ The quadriceps and Achilles tendon reflexes were scored according to the myotatic reflex scale $(M R S)^{15}$ from 0 (reflex absent) to 4 (reflex enhanced), with 2 and 3 corresponding to reflex in the lower and upper half of normal range respectively. The plantar response was graded as flexor (3), mute (2), equivocal (1), or extensor $(0) .{ }^{16}$ In patients with ALS and paraparetic patients, the average score of the right and left lower limb was calculated for each of the above clinical signs. In the case of hemiparetic patients, only the scores of the affected limb were considered for further analysis.

\section{ANALYSIS OF POSTURE}

Body sway during quiet stance was recorded by means of a dynamometric platform (Kistler model 9281B). Patients stood upright barefoot with eyes open (EO) gazing at a target placed at a distance of $50 \mathrm{~cm}$ at eye level, and with eyes closed (EC). Feet were parallel, spaced $10 \mathrm{~cm}$ apart. Two trials, of 52 seconds duration each, were recorded for each visual condition. The forces acting on the platform were acquired and the changes in the position of their resultant vector (the instantaneous CFP) were measured according to the methods described in a previous paper. ${ }^{8}$ From the instantaneous positions of the CFP, the mean position of the CFP was calculated. The mean CFP on the anterior-posterior (A-P) axis in reference to the calcaneum was expressed as a percentage of the total foot length to normalise all the subjects' data. Position of the CFP along the mediolateral axis (CFP-ML) was also calculated: negative and positive values ( $\mathrm{mm}$ ) corresponded to right and left shift of CFP, respectively, with regard to the A-P axis of symmetry. To normalise the subjects' data, the CFP-ML was expressed as a percentage of body height. The body sway area was the surface $\left(\mathrm{mm}^{2}\right)$ swept during the trial by the line joining the mean CFP to the moving instantaneous CFP. ${ }^{17}$

REFLEX RESPONSES TO PERTURBATIONS

Postural reactions to upright stance perturbations were elicited by sudden rotations of a supporting platform. Upward (toe up) rotations stretched the triceps surae muscle, downward rotations (toe down) stretched the tibialis anterior (TA). Velocity and amplitude of rotation were $50^{\circ} / \mathrm{s}$ and $3^{\circ}$. Surface EMG of the soleus and TA muscles were bilaterally recorded. Raw EMG signals were filtered (100 $\mathrm{Hz}-3 \mathrm{kHz},-6 \mathrm{~dB} /$ octave), amplified ( $\times 10000)$ and fed to a computer (sampling frequency 1 $\mathrm{kHz}$ ). EMG activity was off line full wave rectified, and then averaged over 10-20 corresponding postural perturbations. EMG responses were measured with a cursor on the computer screen. The responses in the 
stretched muscles were classified as short latency (SLR) or medium latency response (MLR) when their onset latency was shorter or longer than $60 \mathrm{~ms}$, respectively. The onset of either response was detected at the rise of the EMG signal above 2 SDs of the mean level of its prestimulus background activity, averaged for $100 \mathrm{~ms},{ }^{18}$ and the end of the SLR corresponded to the time at which the EMG signal decreased to below the same level. The area of the SLR was measured between the onset and the end of each response. The area of the soleus and TA MLRs was calculated for a window lasting 45 and $65 \mathrm{~ms}$ after their onset, respectively. The same procedures were used in normal subjects and patients. If the onset of EMG activity occurred at latency longer than 2 SDs above the average onset of the response in normal subjects, the response latency was not considered; the area of the EMG activity was however measured according to the above temporal windows. Because the prestimulus background activity in the soleus and TA muscle was not negligible, and might lead to overestimation of the size of the responses evoked by the perturbation, it was subtracted from the EMG responses. To do this, the area of the background activity was measured over $100 \mathrm{~ms}$ before platform movement, divided by 100 and multiplied by the average duration of the response of normal subjects $(22,17,45$, and 65 ms for soleus SLR, TA SLR, soleus MLR, and TA MLR, respectively).

\section{STATISTICAL ANALYSIS}

All values in the text and table 1 are expressed as grand means (SD) of all subjects and patients. One way analysis of variance (ANOVA) was used to assess differences in clinical findings between the patient groups. The findings obtained in the stabilometric tests were analysed separately according to the visual conditions (EO, EC). Two trials for each condition were considered for all subjects. As body sway area was not normally distributed, normalisation was obtained by logarithmic transformation. Two way ANOVA (with patient groups as the independent variable and visual conditions as the repeated measures) was performed for all sway variables. For reflex responses to perturbations, one way ANOVA was used for assessing differences between the sample of normal subjects and the three groups of patients. For each response, subjects entered the analysis with two observations corresponding to the right and left leg. For hemiparetic patients, the responses from the right and left leg were pooled according to the affected and healthy side. An $F$ value leading to $\mathrm{p}<0.05$ was

Table 1 Mean (SD) scores for clinical findings in patients

\begin{tabular}{lrrr}
\hline Type of assessment & ALS patients & \multicolumn{1}{c}{$\begin{array}{l}\text { Paraparetic } \\
\text { patients }\end{array}$} & $\begin{array}{l}\text { Hemiparetic } \\
\text { patients } \\
\text { (affected leg) }\end{array}$ \\
\hline $\begin{array}{l}\text { Muscle tone of foot plantarflexor muscles } \\
\text { (modified Ashworth scale): 0-5 }\end{array}$ & $1.3(1.4)$ & $2.6(1.4)$ & $1.4(1.2)$ \\
Foot dorsiflexor muscles (MI): 0-33 & $25.4(9.1)$ & $23.0(8.2)$ & $19.5(4.1)$ \\
Quadriceps tendon reflex (MRS): 0-4 & $3.1(0.7)$ & $3.4(0.8)$ & $3.4(1.1)$ \\
Achilles tendon reflex (MRS): 0-4 & $3.0(0.8)$ & $3.2(0.8)$ & $3.4(0.6)$ \\
Plantar response (Norris scale): 0-3 & $1.3(1.2)$ & $0.8(1.0)$ & $0.9(1.3)$ \\
\hline
\end{tabular}

$\mathrm{MI}=$ Motricity index; $\mathrm{MRS}=$ myotatic reflex scale . considered significant. The post hoc test was the Newman-Keuls test. In hemiparetic patients, the area of the responses occurring in the affected and healthy limb were compared through the paired $t$ test. The mean frequency of occurrence of the reflex responses to perturbation was calculated for all groups. For hemiparetic patients, frequency of occurrence was separately calculated for the affected and healthy leg. Any difference in the frequency of occurrence of each response between normal subjects and patients was assessed with a $\chi^{2}$ test.

\section{Results}

\section{CLINICAL FINDINGS}

Muscle tone, strength, tendon reflexes, and plantar response were clinically assessed in the lower limbs of all patients (table 1). On average, muscle tone was increased in all of them. One way ANOVA showed that muscle tone was significantly $(F=5.1, \quad \mathrm{df}=2,49$, $\mathrm{p}<0.01$ ) different between ALS, paraparetic, and hemiparetic patients. The post hoc test showed a significantly greater degree of muscle tone in paraparetic patients than in either patients with ALS (Newman-Keuls, p<0.02) or the affected limb of hemiparetic patients (Newman-Keuls, $\mathrm{p}<0.01$ ). Despite the large between group difference in disease duration, the severity of the other clinical signs was not statistically different between the three groups of patients, thus suggesting that the severity of the disease affecting the motor abilities was homogeneous within each group of patients.

\section{CHARACTERISTICS OF POSTURE}

Sway area

Figure 1 shows an example of body sway recorded during quiet stance under $\mathrm{EO}$ and EC conditions in a normal subject, and in representative patients affected by ALS, paraparesis, or left hemiparesis. The hemiparetic patient swayed to a larger extent than the normal subject, the patient with ALS or the paraparetic patient. The CFP of the three patients was displaced slightly forwards with respect to the normal subject. In addition, in the hemiparetic patient the CFP was shifted toward the unaffected limb.

Figure 2 summarises the results of body sway variables in the normal subjects and in the three patient groups standing quietly under both EO and EC conditions. The graph shows that sway area was within normal limits in patients with ALS and paraparetic patients, but was greatly increased in hemiparetic patients. A two way ANOVA performed on body sway area showed a significant difference between groups $(F=9.3, \mathrm{df}=3,105, \mathrm{p}<0.0001)$ and between visual conditions $(F=113.8, \mathrm{df}=1, \quad 105$, $\mathrm{p}<0.0001)$. A significant $(\mathrm{p}<0.02)$ interaction was found between groups and visual conditions, indicating that closing the eyes did not increase body sway area to the same proportion in all groups. The post hoc test showed that the body sway area of patients with ALS and paraparetic patients was not significantly different from that of normal subjects, whereas the body 
Normal subject

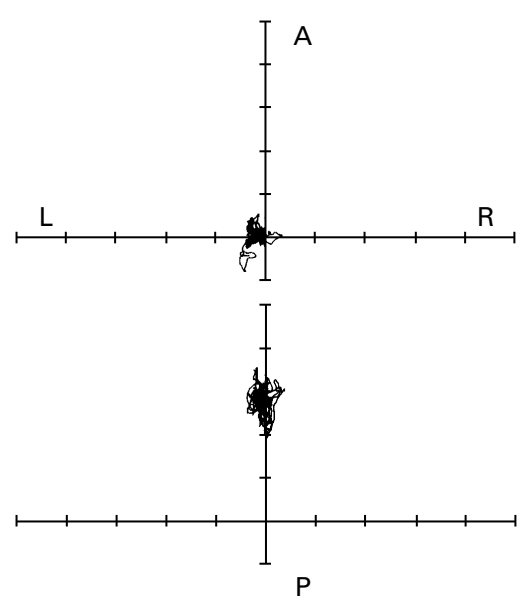

Paraparetic patient
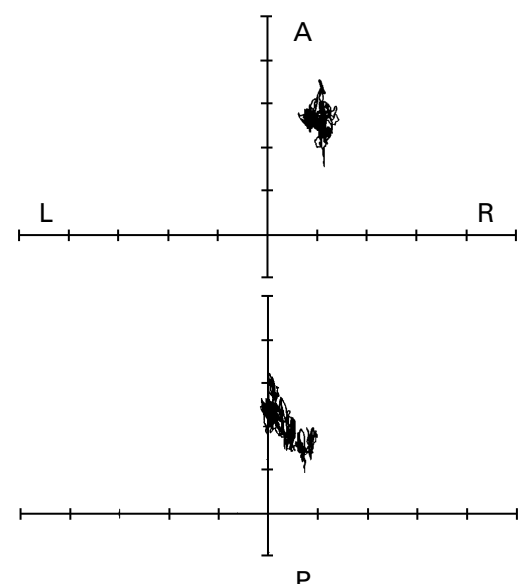

EO

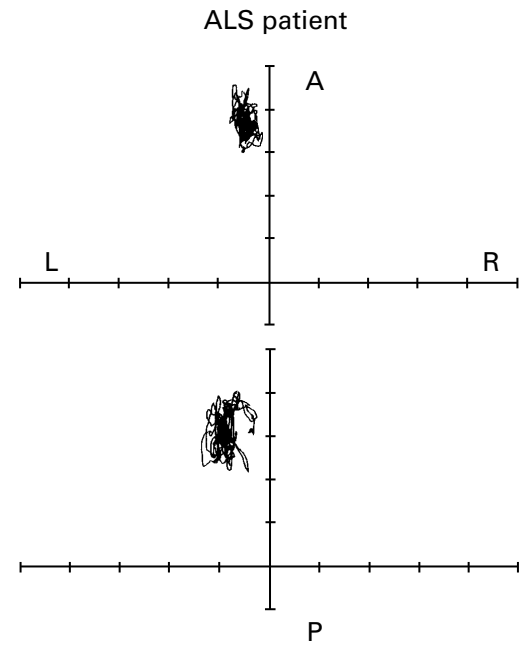

EO

Hemiparetic patient (left)

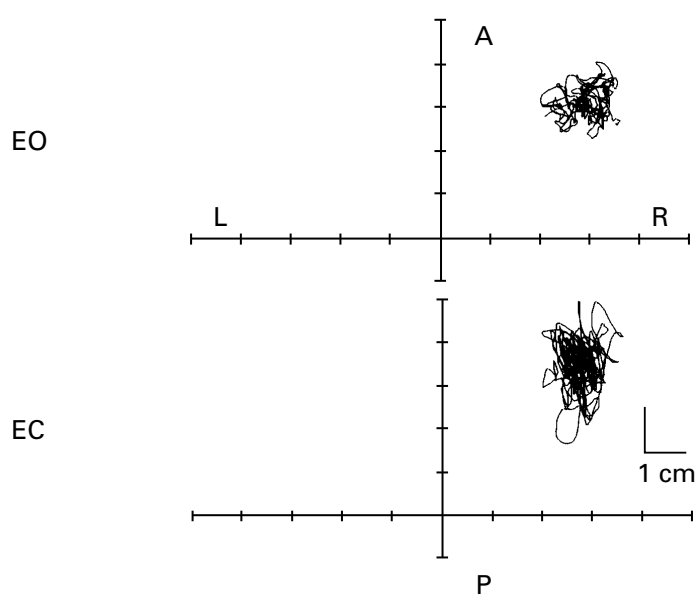

Figure 1 Example of stabilometric recordings obtained during quiet stance with eyes open (EO) and eyes closed (EC) in a normal subject, a patient with amyotrophic lateral sclerosis $(A L S)$, a paraparetic patient, and a hemiparetic patient. All patients showed an anterior shift of the centre of foot pressure (CFP) with respect to the normal subject (for reference, the average CFP along the anterior-posterior axis during the trial obtained in the normal subject under the EO condition was equated to zero). The paraparetic and in particular the hemiparetic patient (left side affected) showed also a lateral shift of the CFP. In the hemiparetic patient, body sway was larger than that in the normal subject and the patients under both visual conditions. $A=$ anterior; $P=$ posterior; $L=$ left; $R=$ right.

sway area of hemiparetic patients was significantly larger (Newman-Keuls, $\mathrm{p}<0.0002$ ) under both visual conditions.

\section{Centre of foot pressure}

Under both visual conditions, the position of CFP A-P (in \% of foot length) was shifted significantly forward $(\mathrm{p}<0.0002)$ in all groups of patients with respect to normal subjects (figure 2 , middle graph). A two way ANOVA on the position of the CFP along the A-P axis showed significant differences between groups $(F=6.8$, $\mathrm{df}=3,105, \mathrm{p}<0.0005)$ but not between visual conditions. There was a moderate forward shift in the paraparetic (Newman-Keuls, $\mathrm{p}<0.002)$ and hemiparetic patients (Newman-Keuls, $\mathrm{p}<0.05)$ with respect to the other groups. For position of the CFP along the M-L axis (in \% of subject height), paraparetic patients showed a slightly greater lateral shift of CFP than the normal subjects and patients with ALS (Newman-Keuls, p<0.05). In hemiparetic patients, the CFP was most affected: these patients had a significantly greater (NewmanKeuls, $\mathrm{p}<0.0002$ ) lateral shift than all other groups, under both visual conditions. Any effect of the A-P or M-L position of the CFP on sway area was assessed. When all patient data were collapsed, the regression between these variables was significant because of the hemiparetic group mean effect; however, within no patient group, hemiparetic patients included, was any relation between body sway area and CFP found. To better weigh up the influence, if any, of M-L position of the CFP on sway area, we compared sway in a subgroup of ALS plus paraparetic patients with respect to a subgroup of hemiparetic patients. The two groups (consisting of eight patients each) had the same M-L CFP. In the ALS plus paraparetic and in the hemiparetic group, the M-L CFP was respectively $1.36 \%(0.29)$ and $1.39 \%(0.29)$ with EO, and $1.35 \%(0.31)$ and $1.05 \%(0.5)$ with EC. Despite the same M-L CFP values in the two groups, the corresponding sway area was significantly $(p<0.005$, 

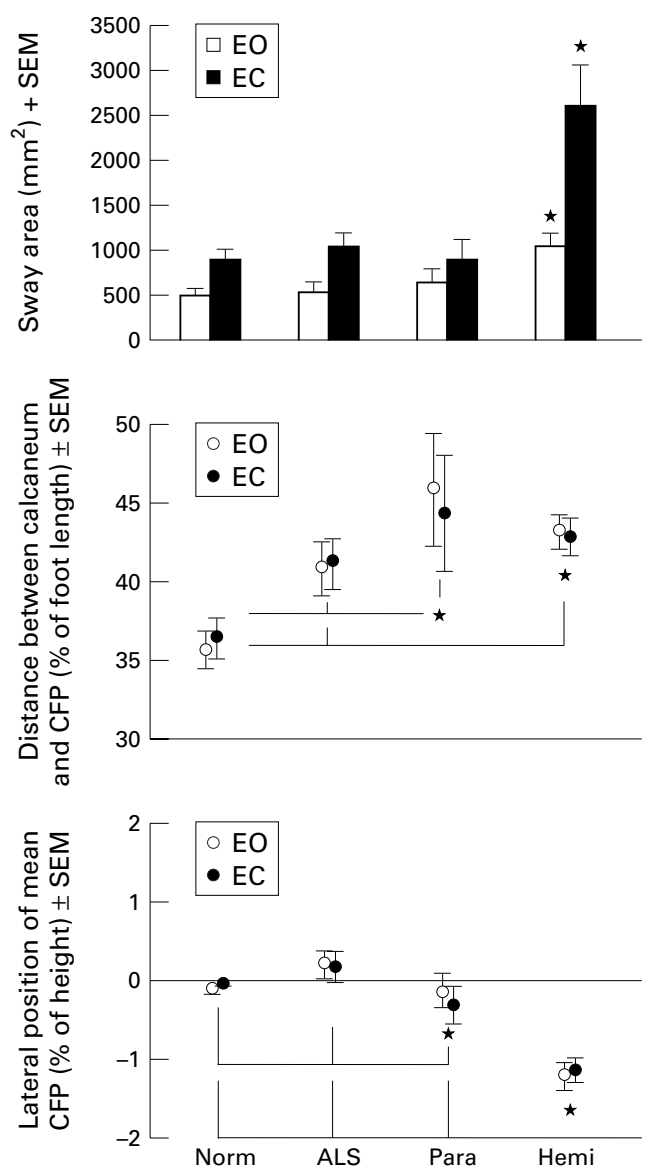

Figure 2 Characteristics of posture in normal subjects and all patients under EO and EC conditions. Each symbol represents the mean (SEM) of body sway area (upper graph), distance between the calcaneum and CFP in \% of foot length (middle graph), and lateral shift of CFP in \% of subject height (lower graph). Norm=normal subjects; ALS = amyotrophic lateral sclerosis; Para $=$ paraparetic; Hemi=hemiparetic. Note the increased forward position of CFP in all patient groups with respect to normal subjects, and the larger lateral shift of CFP in paraparetic and particularly in hemiparetic patients. The hemiparetic group shows the largest increase in sway area under both visual conditions.

unpaired $t$ test) larger in the hemiparetic patients, under both visual conditions. With EO, sway area was $346.0 \mathrm{~mm}^{2}(251.7)$ and $891.2 \mathrm{~mm}^{2}$ (461.0), with EC $800.9 \mathrm{~mm}^{2}$ (801.6) and $2107.8 \mathrm{~mm}^{2}$ (1167.1), respectively in the case of patients with ALS plus paraparetic and hemiparetic patients. It should be noted that, when some of the normal subjects voluntarily leant towards one leg, such as to reproduce the CFP shift seen in hemiparetic patients, the sway area was only slightly increased with respect to the usual upright stance. Therefore, neither uneven distribution of body weight between the two limbs nor body leaning was the sole cause of abnormal body sway.

\section{Relation between stabilometric and clinical findings}

A two way ANOVA was performed between stabilometric variables and clinical variables as muscle tone, strength, tendon reflexes, plantar response, and duration of disease, separately considered. Interestingly, no effect on sway area was exerted by the characteristics of the plantar response: (a) within each group there was no relation between type of plantar response and amplitude of sway; (b) by collapsing all patients' sway in four categories on the basis of the type of response (flexor, mute, equivocal, extensor), no significant or quasi-significant differences were seen. No relation was found between sway or CFP position variables (recorded with EO or EC) and clinical findings in any group of patients. Neither the side (right or left) nor the main localisation of the lesion (frontal, parietal, or frontotemporoparietal) affected the sway variables. In particular, in hemiparetic patients, no change in severity was found in either body sway area or CFP position in the A-P or M-L direction when the patients were divided into two groups on the basis of the side of the lesion. Duration of disease did not affect stabilometric findings, suggesting that the severity of disease was homogeneous within each group of patients.

\section{REFLEX RESPONSES TO PERTURBATION}

Postural muscle background activity

The tonic EMG activity of the soleus and TA muscles of both legs was measured in the 100 ms period before the platform perturbation in all subject groups. The area of this activity was not significantly different (one way ANOVA) either between muscles or subject groups. However, the background activity was larger in the healthy than the affected limb of hemiparetic patients, for both soleus and TA muscles (paired $t$ test $\mathrm{p}<0.05$ ).

\section{NORMAL SUBJECTS}

In normal subjects, the pattern of EMG responses of the leg muscles stretched by toe up or toe down rotation of the supporting platform was similar to that already described in previous papers from our laboratory. ${ }^{19}{ }^{20}$ Figure 3 shows an example of the responses recorded from soleus and TA muscles during the perturbations in the same normal subject and patients depicted in figure 1. In toe up perturbation (stretching triceps surae muscle), the normal pattern consisted of an SLR in soleus muscle, occurring at about $44 \mathrm{~ms}$ in all subjects, followed by a slightly less consistent (frequency of occurrence $90 \%$ ) MLR in the same muscle at about $72 \mathrm{~ms}$. In toe down perturbation (stretching the TA muscle), SLR was infrequently (19\%) evoked in the stretched TA. MLR was instead evoked in TA muscle in $100 \%$ of these subjects, at about 75 ms latency.

\section{PATIENTS}

Frequency of occurrence of EMG responses

The frequency of occurrence of soleus SLR was similar to that of normal subjects, being $96 \%$ in patients with ALS and $100 \%$ in paraparetic and in both limbs of hemiparetic patients. Changes in spastic patients featured the occurrence of a TA SLR to toe down rotation (fig 3). The TA SLR, which was seldom present in normal subjects, occurred with a frequency of $54 \%$ and $86 \%$ respectively in patients with ALS and spastic paraparesis. In 

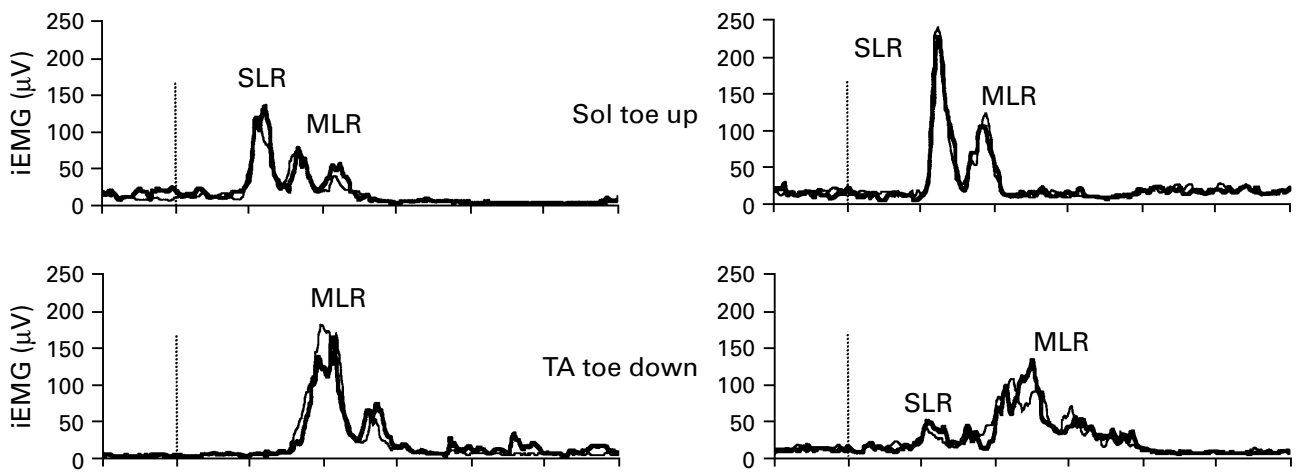

Paraparetic patient

Hemiparetic patient (left)
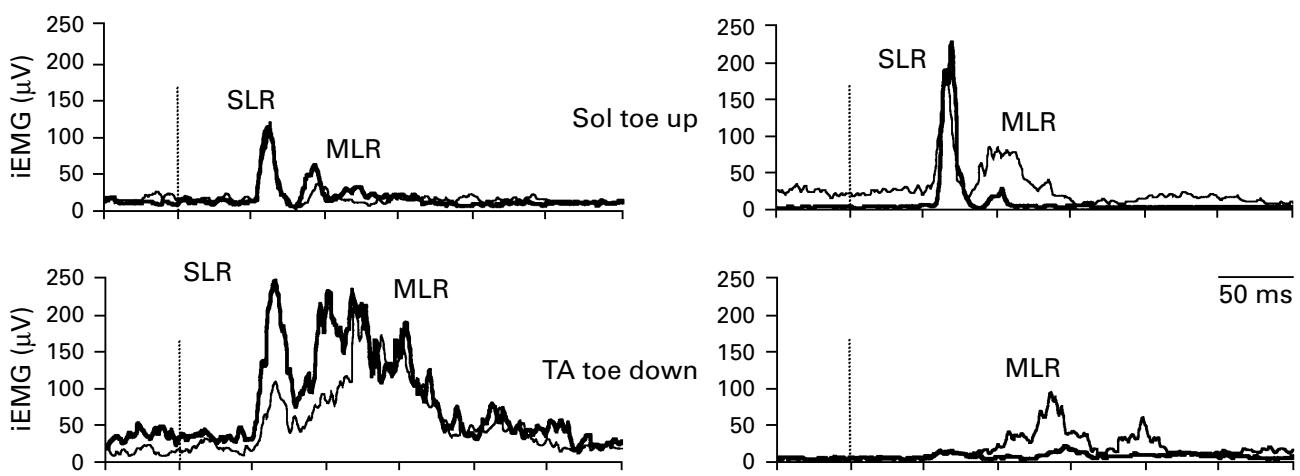

Figure 3 Example of recording of EMG responses of soleus (sol) and tibialis anterior (TA) muscles induced by toe up and toe down rotations of the supporting platform in a normal subject, a patient with ALS, a paraparetic, and a left hemiparetic patient. $S L R=$ short latency response; $M L R=$ medium latency response. In all subjects, each panel shows the superimposition of the average of 20 rectified and filtered (time constant $=1 \mathrm{~ms}$ ) EMG responses from the right (thin line) and left leg (thick line), or affected and non-affected leg for the hemiparetic patient. Note the increased amplitude of the sol SLR in the patients with respect to the normal subject, and the occurrence of the TA SLR in patients with ALS and paraparetic patients. At variance with soleus SLR and TA SLR, soleus MLR and TA-MLR are decreased in amplitude in the affected limb of the hemiparetic patient. Vertical dotted lines indicate the onset of platform displacement.

those with spastic paraparesis, this frequency was significantly $\left(\chi^{2}\right)$ higher than that in normal subjects, patients with ALS and hemiparetic patients. In the affected and healthy limb of hemiparetic patients, the TA SLR occurred respectively at a frequency of $29 \%$ and $21 \%$, neither frequency being significantly different from that in normal subjects.

The frequency of occurrence of the soleus MLR was not significantly different from that of normal subjects, being $92 \%, 93 \%$, and $93 \%$, respectively in patients with ALS, paraparetic patients, and in the affected leg of hemiparetic patients. On the contrary, the frequency of TA MLR was significantly $(\mathrm{p}<0.01)$ decreased in all patient groups compared with normal subjects, being $63 \%, 86 \%$, and $71 \%$, respectively in patients with ALS, paraparetic patients, and in the affected leg of hemiparetic patients. In the healthy leg of the hemiparetic patients, TA MLR occurred with a frequency of $100 \%$, a value significantly higher than that in the contralateral limb.

Latency and area of EMG responses

The latency of the TA-SLR was about 47 ms - that is, similar to that of the soleus SLRthus suggesting that both responses are monosynaptic or oligosynaptic in nature. The latency of the other responses was not significantly affected (fig 3) either, although in the case of hemiparetic patients latency of soleus MLR and TA MLR was increased to a nonsignificant extent in the affected leg with respect to the healthy leg: soleus MLR was delayed by 1.3 (5.3) ms, TA MLR by 7.2 (14.0) ms. Abnormalities in size (fig 4) were found in both limbs of the patients except for the healthy limb of hemiparetic patients, which showed EMG responses similar to those of normal subjects.

The soleus SLR corresponds to the monosynaptic stretch reflex, ${ }^{20}$ and its increased excitability in patients $(F=5.0, \mathrm{df}=3,91, \mathrm{p}<0.005)$ is a well known sign of spasticity. ${ }^{7}$ On average, the area of soleus SLR to toe up rotation was enlarged (Newman-Keuls, $\mathrm{p}<0.05$ ) in patients with ALS and paraparetic patients and marginally $(p<0.06)$ so in the hemiparetic patients with respect to normal subjects. The soleus SLR was significantly larger (paired $t$ test, $\mathrm{p}<0.05)$ in the affected than in the healthy leg of hemiparetic patients.

The area of soleus MLR showed minor and non-specific differences between groups $(F=2.5, \mathrm{df}=3,33, \mathrm{p}<0.07)$. In hemiparetic patients, the soleus MLR was marginally 

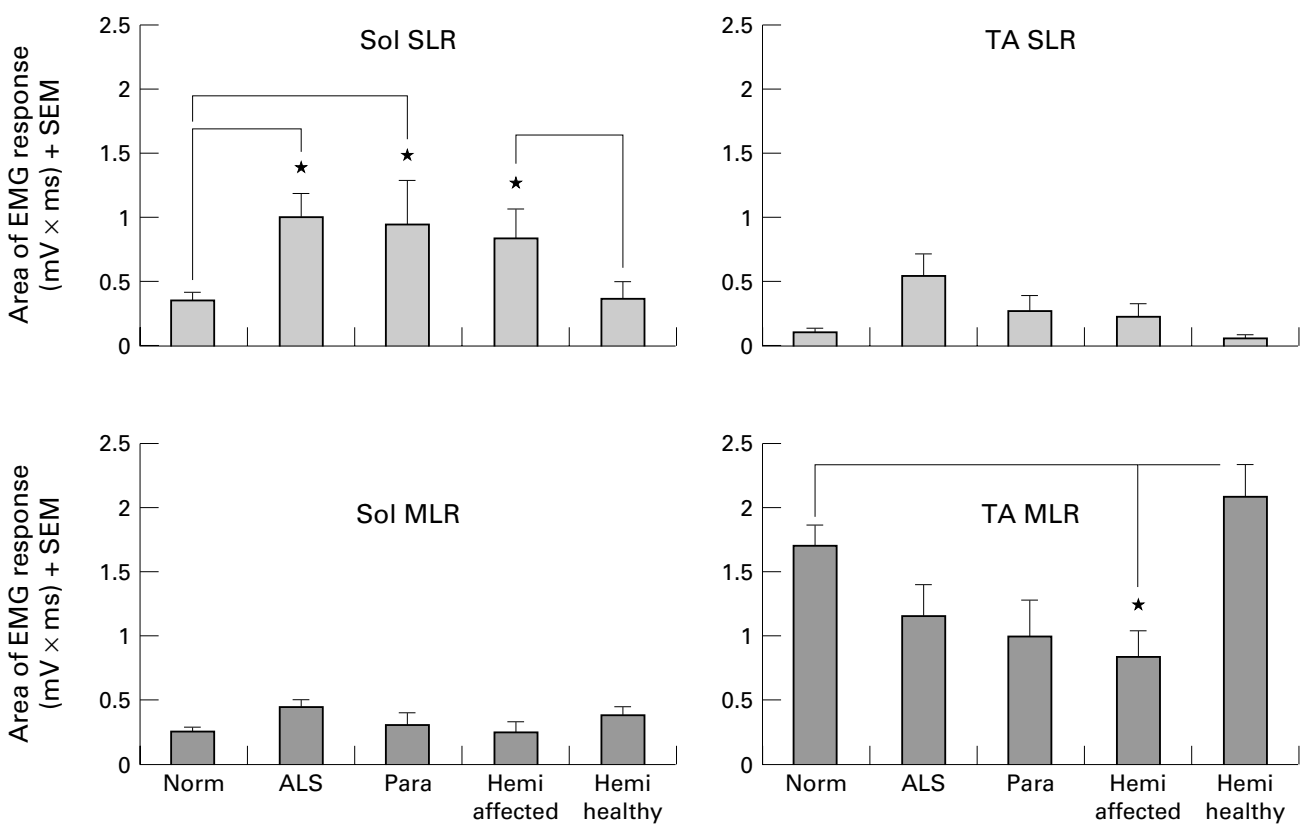

Figure 4 Size of soleus SLR and TA SLR (upper panels) and of soleus MLR and TA MLR (lower panels). From left to right, the bars show the average (+SEM) of the area of the relevant response obtained from normal subjects, patients with ALS, paraparetic, and hemiparetic patients (both affected and healthy side). Note the increased area of soleus SLR in the affected side of hemiparetic patients and particularly so in the patients with ALS and paraparetic patients with respect to normal subjects, and the occurrence of larger than normal TA SLR in the patients. The area of soleus MLR is decreased in hemiparetic patients (affected side). The area of TA MLR is decreased in all patients, and to a larger extent in the affected side of hemiparetic patients. For explanations see figures 2 and 3.

(paired $t$ test, $\mathrm{p}<0.15$ ) decreased in the affected limb with respect to the healthy limb.

The area of TA MLR was significantly $(F=3.7, \mathrm{df}=3,92, \mathrm{p}=0.01)$ different between subject groups. It was decreased in the affected limb of hemiparetic patients with respect to both the healthy limb (paired $t$ test, $\mathrm{p}<0.005$ ) and the normal subjects (Newman-Keuls, $\mathrm{p}<0.05)$. In patients with ALS and paraparetic patients, the TA MLR was reduced only to a marginally significant extent (Newman-Keuls, $\mathrm{p}<0.10$ ) with respect to normal subjects.

A significant positive relation between background EMG activity of soleus muscle and area of the SLR and MLR was found in normal subjects. This was true particularly when soleus responses with respect to soleus background were considered: soleus SLR $\left(\mathrm{y}=0.32 \mathrm{x}+0.20, \mathrm{p}=0.001, R^{2}=0.22\right)$, soleus MLR ( $\left.=0.42 \mathrm{x}+0.13, \mathrm{p}<0.003, R^{2}=0.20\right)$. In patients, despite the large variability in the responses, the regression between the relevant background and area of the soleus SLR was maintained and reached significance in paraparetic patients $(\mathrm{y}=3.80 \mathrm{x}-0.71, \mathrm{p}<0.0001$, $\left.R^{2}=0.75\right)$ and the affected leg of hemiparetic patients $\left(\mathrm{y}=1.77 \mathrm{x}+0.03, \mathrm{p}<0.03, R^{2}=0.35\right)$. Conversely, there was no significant regression between the background and the area of soleus MLR, except for the healthy limb of hemiparetic patients $(\mathrm{y}=0.45 \mathrm{x}+0.16, \mathrm{p}<0.003$, $\left.R^{2}=0.59\right)$.

RELATION BETWEEN STABILOMETRIC FINDINGS AND REFLEX RESPONSES TO POSTURAL PERTURBATION

Much as for the absence of a relation between body sway and clinical variables, we did not find any relation between sway and background
EMG responses in any group. As far as the responses to perturbation are concerned, despite the said changes in the pattern of short and medium latency responses in patients, there was no relation between body sway area, with $\mathrm{EO}$ or $\mathrm{EC}$, and the size of the various responses to perturbations, across all patients or within any group. In particular, sway was not related to the increased amplitude of the SLRs, either in soleus or TA. By generalisation, this finding suggests that the marked increase in body sway in hemiparetic patients could not be explained by the increased excitability of the stretch reflex pathways in postural muscles. For the MLRs, there was no relation between MLR area of either soleus or TA and sway area in any patient group.

\section{Discussion}

PATIENTS WITH ALS AND PARAPARETIC PATIENTS The results show that body sway area is little affected in spastic ALS and paraparetic patients without sensory deficit. The body sway area was not increased in these patients with respect to normal subjects, under either $\mathrm{EO}$ or EC conditions.

No changes or a minor lateral shift in the M-L position of the CFP were found in patients with ALS or paraparetic patients. In both groups, the slightly anterior position of the CFP showed that patients maintained a forward inclined position of the body. Changes in the forward or backward leaning of the body modulates the body sway area in normal subjects. ${ }^{8}$ However, this forward shift was evidently insufficient in patients with ALS and paraparetic patients to produce any effect on body sway area. If anything, the forward sway was simply the counterpart of the increased 
soleus muscle tone, as the higher the tone of the posterior leg muscles the larger the tolerated body forward inclination: a greater stiffness of the postural leg muscles is necessary and can be sufficient to counteract the gravity torque under conditions of body forward leaning. In these patients, the increased stiffness may be attributable to passive rather than active mechanisms, ${ }^{21}$ as the background soleus EMG was in fact not different from normal.

In most patients with ALS and paraparetic patients, there was increased amplitude of short latency stretch responses. This was not related to any increased level of motor pool drive, because, as discussed earlier, background EMG was normal. The increased excitability of the monosynaptic reflex arc was generalised to both extensor and flexor muscles, so that in several of these patients a sizeable SLR was also evoked by toe down rotation in the stretched TA. Therefore, the abnormal excitability of the short latency stretch reflex of leg muscles can fit well with normal body sway. This was true also for the abnormal clinical findings such as increased muscle tone and tendon reflexes, decreased muscle strength, and abnormal plantar response. It seems then that spasticity and some of its major sequelae do not lead to poor control of quiet upright stance. The medium latency response, the size of which is an indication of the excitability of the pathway originating in the spindle secondary afferents, ${ }^{22}$ was present in many of these patients both on soleus and TA muscles and was, on average, of normal amplitude or diminished.

\section{HEMIPARETIC PATIENTS}

At variance with patients with ALS and paraparetic patients, hemiparetic patients showed significantly larger body sway values under both $\mathrm{EO}$ and $\mathrm{EC}$ conditions. At variance with previous reports, ${ }^{23}{ }^{24}$ the increase in body sway was not connected to the side (right or left) or the main localisation (cortical or subcortical) of the lesion. ${ }^{11}$ This discrepant finding might be explained by the exclusion of patients with sensory deficits from our study and by the absence of lesions in the right posterior parietal cortex, known to be involved in the processing of internal spatial representation. ${ }^{1125}$ In our hemiparetic patients, sway increase was accompanied by a significant forward shift of CFP, greater than in normal subjects but similar to that of paraparetic patients. However, as shown for patients with ALS and paraparetic patients, the occurrence of either spasticity or forward shift of the CFP cannot explain an increase of body sway area. At variance with the other patients, hemiparetic patients showed a lateral shift of CFP, a sign of the body weight being unevenly distributed between the lower limbs. In normal subjects, however, voluntary displacement of the body, such as to shift the CFP to the same extent as in hemiparethic patients, resulted in a much slighter increase in body body sway area (unpublished observation). The idea that the increase in body sway area found in hemiparetic patients is connected with the eccentric position of the CFP is further weakened by the absence of a relation between sway area and CFP shift within the group, and by a normal body sway area in those patients with ALS and paraparetic patients whose CFP was shifted laterally to the same extent as in hemiparetic patients. In conclusion, even in the hemiparetic patients, the increased body sway area cannot be solely explained by their faulty posture, although it is conceivable that stance control may be partly worsened by the shift of the body weight toward the healthy limb. ${ }^{2} 2627$

The pattern of EMG reflex responses to postural perturbations in the affected leg of hemiparetic patients was unlike that of the others. The short latency response to stretch was increased, but not to any greater degree than in the other spastic patients. Conversely, the medium latency responses of soleus and TA were reduced, in the affected leg, more than in the other groups. The responses to stretch, which are mediated by the spindle group II afferent fibres, ${ }^{22}$ are evidently subjected in hemiparesis to a different descending control from that acting on the short latency response. ${ }^{128}$ This notion is strengthened by the finding of no relation between background soleus EMG and amplitude of soleus MLR, whereas a positive relation between these two variables is present in normal subjects and in the healthy limb of the hemiparetic patients. One important source of increased sway in hemiparetic patients can therefore be the decreased modulation in excitability of this response to stretch in the affected limb. There are in fact suggestions that it is this medium latency response, rather than the monosynaptic reflex, that plays a part in the reflex control of quiet stance. ${ }^{27}{ }^{29}$ This notion is based of course on the assumption that stance support by the affected limb is not negligible despite the body shift toward the healthy leg and, therefore, that the abnormal reflex contribution from the affected limb has a detrimental effect on upright stance control. Notably, recent data show that the increase in body sway during stance in hemiparetic patients can be connected with asymmetric temporal synchronisation of postural oscillation between the two legs. ${ }^{30}$

\section{Conclusion}

Since neither body attitude, nor paresis or monosynaptic reflex hyperexcitability seem to affect body sway, it can be hypothesised that decreased muscle force or abnormal processing of the input from primary spindle endings or foot sole cutaneous receptors are not paramount in the control of equilibrium during quiet upright stance. Conversely, although no direct information is given by the experiments, the present findings are in favour of a role of the afferent input from spindle secondary endings in the reflex control of equilibrium. In fact, where the normal modulation of excitability of the reflex pathway fed by spindle group II afferent fibres is hardly affected, as in patients with ALS and paraparetic patients, stance stability is within normal limits; on the contrary, in hemiplegia the diminished medium latency 
response is accompanied by increased body sway. Velocity sensitive spindle primaries would provide a less important input to the nervous system than the length sensitive spindle secondaries, as during quiet stance very slow body movements occur. ${ }^{10}{ }^{31}$ Under this condition, the input from the secondaries should suffice to enable the nervous system to detect low frequency displacements of the body ${ }^{29}$ and produce appropriate changes in muscle tone to counteract the perturbations of balance. ${ }^{32}$ This interpretation is further supported by our recent findings that postural control during quiet stance of patients with peripheral neuropathy ${ }^{29}$ is negligibly affected by loss of group Ia afferent fibres whereas it is so when group II spindle afferent fibres are also involved by the disease.

We are indebted to Drs Letizia Mazzini and Gabriele Mora for referring patients under their care. This research was partly supported by the Italian Ministry of Health ("Ricerca Corrente" grant 1992/96), MURST ("Progetti di Ricerca di Interesse Nazionale" grant 1997 and 1999), and Telethon-Italy (gran Nos 1071C and 1160C). R Allpress scrutinised the English.

1 Dietz V. Human neuronal control of automatic functional movements: interaction between central programs and afferent input. Physiol Rev 1992;72:33-69.

2 Shumway-Cook A, Anson D, Haller S. Postural sway biofeedback: its effect on reestablishing stance stability in hemiplegic patients. Arch Phys Med Rehabil 1988;69:395400 .

3 Mizrahi J, Solzi P, Ring H, et al. Postural stability in stroke patients: vectorial expression of asymmetry, sway activity and relative sequence of reactive forces. Med Biol Eng Comput 1989;27:181-90.

4 Horak FB, Macpherson JM. Postural orientation and equilibrium. In: Rowell LB, Shepherd JT, eds. Exercise: regulation and integration of multiple systems handbook of regulation and integration of multiple systems handbook of physiology. New

5 Nardone A, Miscio G, Pisano F, et al. Sensory ataxia in polyneuropathy is connected to dysfunction of group II afferent fibers. F Peripher Nerv Syst 1998;3:151.

6 Magnusson M, Enbom H, Johansson R, et al. Significance of pressor input from the human feet in anterior-posterio postural control. Acta Otolaryngol (Stock) 1990;110:182-8

7 Burke D. Spasticity as an adaptation to pyramidal tract injury. In: Waxman SG, ed. Advances in neurology. New York: Raven Press 1988;47:401-23.

8 Schieppati M, Hugon M, Grasso M, et al. The limits of equilibrium in young and elderly normal subjects and in parkinsonians. Electroencephalogr Clin Neurophysiol 1994:93. parkinso 286 .

9 Nardone A, Tarantola J, Galante M, et al. Time course of stabilometric changes after a strenuous treadmill exercise. Arch Phys Med Rehabil 1998;79:920-4.

10 Nardone A, Tarantola J, Giordano A, et al. Fatigue effects on body balance. Electroencephalogr Clin Neurophysiol 1997; 105:309-20.
11 Andersen RA, Snyder LH, Bradley DC, et al. Multimodal representation of space in the posterior parietal cortex and its use in planning movements. Ann Rev Neurosci 1997;20: 303-30.

12 Ashworth B. Preliminary trial of carispodol in multiple sclerosis. Practitioner 1964;192:540-2.

13 Bohannon RW, Smith MB. Interrater reliability of a modified Ashworth scale of muscle spasticity. Phys Ther 1987;67:206-7.

14 Collin C, Wade DT. Assessing motor impairment after stroke: a pilot reliability study. $\mathcal{F}$ Neurol Neurosurg Psychiatry 1990;53:576-9.

15 Hallett M. NINDS myotatic reflex scale. Neurology 1993;43: 2723.

16 Norris FH, Calanchini PR, Fallat RJ, et al. The administration of guanidine in amyotrophic lateral sclerosis. Neurology 1974;24:721-8.

17 Diener HC, Dichgans J, Bacher M, et al. Quantification of postural sway in normals and patients with cerebellar disease. Electroencephalogr Clin Neurophysiol 1984;57:13442.

18 Schieppati M, Nardone A. Medium-latency stretch reflexes of foot and leg muscles analysed by cooling the lower limb in standing humans. F Physiol (Lond) 1997;503:691-8.

19 Nardone A, Siliotto R, Grasso M, et al. Influence of aging on leg muscle reflex responses to stance perturbation. Arch Phys Med Rehabil 1995;76:158-65.

20 Schieppati M, Nardone A, Siliotto R, et al. Early and late stretch responses of human foot muscles induced by perturbation of stance. Exp Brain Res 1995;105:411-22.

21 Dietz V, Quintern J, Berger W. Electrophysiological studies of gait in spasticity and rigidity: evidence that altered mechanical properties of muscle contribute to hypertonia. Brain 1981;104:431-49.

22 Rode G, Tiliket C, Boisson D. Predominance of postural imbalance in left hemiparetic patients. Scand $\mathcal{F}$ Rehabil Med 1997;29:11-6.

23 Niam S, Cheung W, Sullivan P, et al. Balance and physical impairments after stroke. Arch Phys Med Rehabil 1999;80: 1227-33.

24 Bonda E, Frey S, Petrides M. Evidence for a dorso-medial parietal system involved in mental transformation of the body. 7 Neurophysiol 1996;76:2042-8.

25 Dickstein R, Nissan M, Pillar T, et al. Foot-ground pressure pattern of standing hemiplegic patients. Major characteristics and patterns of improvement. Phys Ther 1984;64:1923.

26 Dettmann MA, Linder MT, Sepic SB. Relationships among walking performance, postural stability, and functional assessment of the hemiplegic patient. Am f Phys Med 1987; 66:77-90

27 Schieppati M, Nardone A. Group II spindle afferent fibers in humans: their possible role in the reflex control of stance. Progr Brain Res 1999;123:461-72.

28 Corna S, Grasso M, Nardone A, et al. Selective depression of medium-latency leg and foot muscle responses to stretch by an $\alpha_{2}$-agonist in humans. F Physiol (Lond) 1995;484: $803-9$.

29 Nardone A, Tarantola J, Miscio G, et al. Loss of large-diameter spindle afferent fibres is not detrimental to the control of body sway during upright stance: evidence from neuropathy. Exp Brain Res 2000;135:155-62.

30 Dickstein R, Abulaffio N. Postural sway of the affected and non-affected pelvis and leg in stance of hemiparetic patients. Arch Phys Med Rehabil 2000;81:364-7.

31 Gurfinkel EV. Physical foundations of stabilography. Aggressologie 1973;14:9-13.

32 Morasso P, Schieppati M. Can muscle stiffness alone stabilize upright standing? $\mathcal{F}$ Neurophysiol 1999;83:1622-6. 